\title{
Dolor miofascial lumbar en estudiantes de Terapia Física y Rehabilitación de la Universidad Nacional Federico Villarreal, 2012
}

\author{
Cristhian Santiago Bazán
}

\section{RESUMEN}

Objetivo: Conocer las características asociadas a dolor lumbar en estudiantes de pregrado.

Material y Métodos: estudio descriptivo, tipo transversal en 227 alumnos.

Resultados: se observó, una alta prevalencia de dolor lumbar y como factor estadísticamente significativo a la ansiedad y depresión. Así mismo, los alumnos que padecieron dolor lumbar, manifestaron limitaciones en sus actividades académicas, actividades de vida diaria y problemas emocionales.

Conclusiones: estos hallazgos plantean la necesidad de desarrollar programas preventivos en esta población, llevando a cabo evaluaciones ergonómicas en el centro de estudio, programas de ejercicios para mejorar la flexibilización, el arco de movilidad potencia y fuerza muscular mejorando su calidad de vida y competencias propias del ejercicio profesional, con eficiencia en los futuros profesionales de la salud. (Horiz Med 2014; 14(4): 19-23)

Palabras clave: Dolor Lumbar; Ansiedad; Depresión; Terapia Física. (Fuente: DeCS BIREME).

Myofascial lumbar pain in Physical Therapy and Rehabilitation students of Universidad Nacional Federico Villarreal, 2012

\section{ABSTRACT}

Objective: To know the associated characteristics of lumbar pain in undergraduate students.

Material and Methods: Descriptive, crossectional study in 227 students.

Results: we observed a high prevalence of low back pain and statistically significant factor to anxiety and depression. Likewise, students who have had back pain, expressed limitations in their academic activities, activities of daily living and emotional problems.

Conclusions: These findings suggest the need to develop prevention programs in this population, conducting ergonomic assessments at the study center, exercise programs to improve flexibility, range of motion and muscle power to improve their quality of life and competency of professional practice, efficiently in future health professionals. (Horiz Med 2014; 14(4): 19-23)

Key words: Back Pain, Risk Factors, Anxiety, Depression, Physical Therapy. (Source: MeSH NLM).

1 Tecnólogo médico en terapia física y rehabilitación. Magister en Gestión y Conducción en Salud. Profesor de la Universidad Privada San Juan Bautista. 


\section{INTRODUCCIÓN}

El dolor lumbar es un problema de Salud Pública, que se ve con mayor frecuencia en la consulta a Nivel mundial, que podría conllevar a diferentes grados discapacidad, afectando al individuo en su calidad de vida. En el caso de los Estudiantes de Fisioterapia y Rehabilitación, genera ausentismo a clases, afectando su aprendizaje académico.

El dolor lumbar, se da con mayor frecuencia en trabajadores que se someten a esfuerzos físicos pesados y en aquéllos cuya actividad laboral exige posiciones forzadas, incluso una postura estática constante. Así, son causas de riesgo importantes: levantar objetos, agacharse y el doblarse.

La frecuencia de lumbalgia entre trabajadores cuyas tareas implican levantar grandes pesos es más de ocho veces superior a la de aquéllos que no levantan cargas. El levantar obj etos pesados, según la literatura epidemiológica mundial, es la causa terminal más importante en la aparición de las lumbalgias. El trabaj o físico pesado y la postura forzada sostenida, son los desencadenantes principales de la osteoartrosis, la degeneración discal y el dolor ciático (1).

El factor socio-cultural, tiene mucha importancia en el país, la educación y la experiencia de dolor lumbar se basan en las creencias de dolor de espalda. Las creencias negativas, hacen que se agrave el dolor de espalda (2).

Existe un riesgo mayor en trabajadores de turnos de trabajo para lumbago con ciática y mayor riesgo en trabajadores con puestos de trabajo tipo blue collar, para lumbago sin ciática (3).

La profesión del fisioterapeuta,conlleva a una serie de factores de riesgo para el dolor vertebral, tales como sus características de actividad física (cargas, aplicación de fuerzas, bipedestación estática, etc.), la realización de menos descansos, tanto en el trabajo como fuera de él, la situación laboral inestable, etc., todo ello supone un cúmulo de factores de riesgo para padecer dolor vertebral.

Estos hechos pueden hacernos pensar que el exceso de carga de trabajo extraprofesional, la situación de inestabilidad laboral, así como la falta de descanso, sean los responsables del padecimiento de dolor vertebral de origen psicosomático en las mujeres estudiadas, añadiendo además el factor de riesgo que supone en sí la profesión de fisioterapeuta para el padecimiento de algias vertebrales (4).

El dolor músculo-esquelético, afecta tanto a los profesionales de la salud que laboran en países industrializados con tecnología de punta, como a profesionales de la salud de países en vía de desarrollo. El fisioterapeuta, es el profesional de la salud encargado de curar, prevenir, recuperar y adaptar a la persona a partir de medios físicos.

Paradójicamente, estos profesionales, que brindan cuidados de salud, son susceptibles a lesiones del sistema músculo-esquelético, por las características de su trabajo, a pesar de que poseen el conocimiento anatómico-fisiológico de su cuerpo, principios de mecánica corporal y otros conceptos preventivos que forman parte de su formación.

De igual forma, acontece este hecho en los estudiantes de Fisioterapia.

Esta elevada prevalencia, es fruto de los riesgos de su entorno ocupacional; una inadecuada higiene postural, así como la práctica de trabajos repetitivos y actividades físicas intensivas.

Estos factores, hacen que, dependiendo de los Centros en los que se han realizado las estancias clínicas, y el número de horas prácticas que se han realizado, la prevalencia de lesiones aumente o disminuya en función de las formas y métodos de trabajo.

Pero la verdadera razón que hace que la prevalencia de lesiones aumente entre estos estudiantes, es la falta de cumplimiento de las medidas ergonómicas, pese a tener a su alcance, los conocimientos necesarios para determinar que, el nivel de exigencias físicas impuestas por la tarea que realizan y el entorno donde se desarrolla ésta, no están en la mayoría de los casos dentro de los límites fisiológicos y biomecánicos aceptablesy pueden llegar a sobrepasar lascapacidades físicas de cada persona con el consiguiente riesgo para su salud (5). 


\section{MATERIAL Y MÉTODOS}

Estudio observacional, descriptivo, de corte transversal. Se obtuvo la prevalencia y características de dolor lumbar en los alumnos participantes de Terapia Física y Rehabilitación de una Universidad Estatal, mediante una encuesta de tipo transversal para poder determinar la prevalencia y factores asociados del Dolor Lumbar, la cual se obtuvo con una confiabilidad de 0.71 (Alfa de Crombach) y un coeficiente de correlación $>0.33$ de regular a buena. Se obtuvo mediciones con respecto al Índice de Masa Corporal con ayuda de un tallímetro y una balanza electrónica marca CAMRY. Se aplicó un Test de Ansiedad y Depresión (Test de Golberg).

\section{RESULTADOS}

Se tuvo la participación de 227 estudiantes de Terapia Física, el 66,5\% (151), manifestaron dolor en la región lumbar. Tabla 1.

Se determinó los siguientes factores asociados a Dolor Lumbar: ansiedad con un $p \varangle 0,003$ y depresión, el cual reflejó $\mathrm{p}<0,001$.

De los 151 estudiantes de Terapia Física y Rehabilitación, que padecían dolor lumbar, lo limitaba con mayor relevancia el cargar obj etos pesados, en el $70,9 \%(107)$ y al agacharse, 53,6\% (81). Tabla 2.

Tabla 1. Prevalencia de Dolor Lumbar en los Alumnos de Tecnología Médica de la Universidad Nacional Federico Villarreal en el 2012

\section{Dolor Lumbar}
No
$\mathrm{Si}$
Total

$\begin{array}{lllll}\mathbf{N}^{\circ} & \% & \mathbf{N}^{\circ} & \% & \mathbf{N}^{\circ}\end{array}$

Tabla 2. Desencadenante de dolor lumbar.

\begin{tabular}{|c|c|c|c|c|c|c|}
\hline \multirow{2}{*}{$\begin{array}{l}\text { Limitación de } \\
\text { actividades a } \\
\text { consecuencia del } \\
\text { dolor lumbar }\end{array}$} & \multicolumn{2}{|c|}{ Total } & \multicolumn{2}{|r|}{ Si } & \multicolumn{2}{|r|}{ No } \\
\hline & $\mathbf{N}^{\circ}$ & $\%$ & $\mathbf{N}^{\circ}$ & $\%$ & $\mathbf{N}^{\circ}$ & $\%$ \\
\hline AGACHARSE & 151 & $100.0 \%$ & 81 & $53.6 \%$ & 70 & $46.4 \%$ \\
\hline $\begin{array}{l}\text { CARGAR OBJETOS } \\
\text { LIVIANOS }\end{array}$ & 151 & $100.0 \%$ & 19 & $12.6 \%$ & 132 & $87.4 \%$ \\
\hline $\begin{array}{l}\text { ROTACION DE } \\
\text { TRONCO }\end{array}$ & 151 & $100.0 \%$ & 69 & $45.7 \%$ & 82 & $54.3 \%$ \\
\hline $\begin{array}{l}\text { CARGAR OBJETOS } \\
\text { PESADOS }\end{array}$ & 151 & $100.0 \%$ & 107 & $70.9 \%$ & 44 & $29.1 \%$ \\
\hline $\begin{array}{c}\text { REALIZAR QUE } \\
\text { HACERES } \\
\text { DOMESTICOS (LAVAR, } \\
\text { PLANCHAR, } \\
\text { COCINAR, ETC) }\end{array}$ & 151 & $100.0 \%$ & 54 & $35.8 \%$ & 97 & $64.2 \%$ \\
\hline
\end{tabular}

De los 227 estudiantes de Terapia Física y Rehabilitación, que padecían dolor lumbar, cargaban un peso de siete a nueve kilogramos: $23,7 \%$ (18) y de cuatro a seis kilogramos $32,9 \%$ (25). Tabla 3.

Tabla 3. Carga de peso y dolor lumbar.

\begin{tabular}{|c|c|c|c|c|c|c|}
\hline \multirow{2}{*}{$\begin{array}{l}\text { Carga de peso } \\
\text { en actividades } \\
\text { cotidianas }\end{array}$} & \multicolumn{2}{|r|}{ No } & \multicolumn{2}{|c|}{ Si } & \multicolumn{2}{|c|}{ Total } \\
\hline & $\mathbf{N}^{\circ}$ & $\%$ & $\mathbf{N}^{0}$ & $\%$ & $\mathbf{N}^{0}$ & $\%$ \\
\hline $\begin{array}{l}\text { No más de } \\
\text { un kilogramo }\end{array}$ & 1 & $1.3 \%$ & 4 & $2.6 \%$ & 5 & $2.2 \%$ \\
\hline $\begin{array}{l}\text { De una a tres } \\
\text { kilogramos }\end{array}$ & 16 & $21.1 \%$ & 33 & $21.9 \%$ & 49 & $21.6 \%$ \\
\hline $\begin{array}{c}\text { De cuatro a } \\
\text { seis kilogramos }\end{array}$ & 25 & $32.9 \%$ & 58 & $38.4 \%$ & 83 & $36.6 \%$ \\
\hline $\begin{array}{c}\text { De siente a } \\
\text { nueve kilogramos }\end{array}$ & 18 & $23.7 \%$ & 13 & $8.6 \%$ & 31 & $13.7 \%$ \\
\hline $\begin{array}{l}\text { De } 10 \text { a más } \\
\text { kilogramos }\end{array}$ & 16 & $21.1 \%$ & 43 & $28.5 \%$ & 59 & $26 \%$ \\
\hline Total & 76 & $100 \%$ & 151 & $100 \%$ & 227 & $100 \%$ \\
\hline
\end{tabular}




\section{DISCUSIÓN}

Se encontraron importantes datos epidemiológicos y estadísticos, que servirán para estudios posteriores y desarrollo de Programas y Proyectos de Extensión de Bienestar Social.

Los datos presentados, confirman que existe una elevada prevalencia de dolor lumbar $(66,5 \%)$, en la población estudiantil de Terapia Física y Rehabilitación de la Facultad de Tecnología Médica de la UNFV que es comparable a otros estudios realizados en el mismo grupo poblacional en otros países, como demuestra Camargo, en su estudio con alumnos de Terapia Física y Rehabilitación. La prevalencia del dolor crónico es un asunto de gran importancia para los profesionales de la salud, los sistemas nacionales de salud, los pacientes y la sociedad. Los datos obtenidos difieren unos de otros debido a las variaciones de población de las muestras, los métodos usados para su obtención.

Las características del dolor lumbar, concuerdan con las manifestaciones en diferentes investigaciones (5-10) en las que se demostró una relación directa con los resultados. Es evidente, pues, que el dolor de espalda baja, representa un importante problema, tanto en el ámbito médico, social y, consecuentemente, político, debido a los elevados costos personales y económicos.

En la práctica clínica, las características del dolor lumbar como: intensidad, tiempo de aparición, localización del dolor, frecuencia en que se manifiesta el dolor, son de carácter subjetivo. La cronicidad del dolor, depende no sólo de las características del proceso, sino también de la idiosincrasia del paciente y del manejo que se haga del cuadro, puede afectar diversos aspectos de la vida del paciente, cambiando su estilo de vida, como su estado emocional. Estas manifestaciones, concuerdan en varias investigaciones (7-11), las que tienen una gran similitud con nuestro estudio; no hay duda, que el dolor de espalda baja es de elevada intensidad en un número sustancial de pacientes.

Este trabajo, permite obtener datos propios sobre las características del dolor de espalda en la población general y puede contribuir a una mejor planificación de estrategias sanitarias y a la toma de decisiones políticas que puedan ayudar a disminuir el número de personas afectadas con este problema de salud pública, con énfasis en la comunidad estudiantil universitaria.

La evaluación del estado funcional es esencial en el tratamiento de los pacientes con dolor 0 alteraciones músculo esqueléticas, siendo que el dolor y la incapacidad, son los puntos más importantes para este tipo de población.

Se observó que un grupo importante de los pacientes tuvo limitada su actividad habitual, incluso en actividades cotidianas importantes (al agacharse, cargar objetos livianos, cargar objetos pesados y realizar quehaceres domésticos) (7-9).

Existió relación entre la ansiedad y depresión, factor influyente, tanto en el comportamiento y la eficacia de los diferentes agentes físicos y técnicas de rehabilitación en la recuperación del paciente.

En investigaciones similares, otros autores señalan, relación con nuestros resultados (9). Demostrando una correlación positiva entre síntomas depresivos y dolor lumbar.

Se puede considerar que el dolor y la ansiedad están relacionados. El tratamiento de la ansiedad y dolor a través de la fisioterapia, puede ser efectivo sobre todo, si se combina con otras terapias. El tratamiento multidisciplinario puede ser un recurso terapéutico aconsejable para el abordaje de la comorbilidad dolor-ansiedad. Tanto la lumbalgia crónica como los trastornos de la ansiedad son muy frecuentes en la actualidad; el $60-90 \%$ de las personas han sufrido en algún momento de su vida lumbalgia.

El tratamiento de la ansiedad y dolor a través de la fisioterapia puede ser efectivo sobre todo si se combina con otras terapias.

Las posturas de trabajo muy exigentes por tiempos prolongados como: bipedestación con flexión o torsión constante de tronco, levantamiento constante de cargas ligeras o pesadas, manejo 
constante de materiales manuales, pueden influir en la aparición de procesos dolorosos de la región lumbar. La carga de peso es un factor predisponente en el dolor lumbar (9).

Acerca de los resultados no estadísticamente significativos, existen estudios que hallan relación con nuestros resultados $(9,10-12)$, demostrando una alta correlación positiva entre síntomas depresivos y dolor; en especial, las que fumaban más de 10 cigarrillos diarios (9).

En nuestro país, existe un déficit de estudios relacionados exclusivamente a los alumnos universitarios de Terapia Física y Rehabilitación, con respecto al dolor lumbar a comparación de otros países de Latinoamérica y la Comunidad Europea.

Es necesario implantar programas que den solución a este problema de Salud Pública.

\section{Fuentes de financiamiento}

El estudio ha sido autofinanciado por el autor.

\section{Conflicto de interés}

El autor declara no tener ningún conflicto de interés.

\section{Correspondencia:}

\section{Cristhian Santiago Bazán}

Dirección: Jr. Tiahuanaco 1493 Urb Zárate San Juan de . Lurigancho. Lima - Perú

Teléfono: RPC 941703104

Correo electrónico: cristhiansantiagob@gmail.com

\section{REFERENCIAS BIBLIOGRÁFICAS}

1. Noriega-Elío M, Barrón Soto A, Sierra Martínez O, Méndez Ramírez I, Pulido Navarro M, Cruz Flores C. The debate on lower back pain and its relationship to work: a retrospective study of workers on sick leave. Cad Saúde Pública. 2005;21(3):887-97.

2. Burnett A, Sze CC, Tam SM, Yeung KM, Leong M, Wang WT, et al. A Cross-cultural Study of the Back Pain Beliefs of Female Undergraduate Healthcare Students: Clin J Pain. 2009; 25(1):20-8.

3. Baldeón P, Carlos J, Ruíz Gutierrez F, Navarro Chumbes G, Dongo Lazo F, Llap Yesan C, et al. El trabajo a turnos como factor de riesgo para lumbago en un grupo de trabajadores peruanos. Rev Medica Hered. 2005 Jul;16(3):184-9.

4. Simon M, Tackenberg $P$, Nienhaus A, Estryn-Behar M, Maurice Conway P, Hasselhorn H-M. Back or neck-pain-related disability of nursing staff in hospitals, nursing homes and home care in seven countries-results from the European NEXT-Study. Int J Nurs Stud. 2008 Jan;45(1):24-34.

5. Muela-Dueñas P. Asociación entre las lesiones músculo esqueléticas y los riesgos ergonómicos en los alumnos del segundo y tercer curso de la Escuela de Fisioterapia de Cantabria: ison los fisioterapeutas víctimas de su propio dogma? [Internet]. [citado 2012 Set 12]. Disponible en: http://www.efisioterapia.net/articulos/asociacionlas-lesiones-musculo-esqueleticas-y-los-riesgos-ergonomicos-losalumnos-del-seg

6. Moreno Lorenzo N, Pineda Galán C, Díaz Mohedo E, Barón López FJ, Sánchez Guerrero E, Labajos Manzanares MT. Estudio transversal de las algias vertebrales en los fisioterapeutas. Fisioterapia. 2003;25(1):23-8.

7. Camargo Lemos DM, Orozco Vargas LC, Hernández Sánchez J, Niño Cruz Gl. Dolor de espalda crónico y actividad física en estudiantes universitarios de áreas de la salud. Rev Soc Esp Dolor. 2009 Nov;16(8):429-36.

8. Rosero-Martínez RV, Vernaza-Pinzón P. Perfil postural en estudiantes de fisioterapia. Aquichan [Internet]. 2010 [citado 2012 Oct 12];10(1). Disponible en: http://aquichan.unisabana.edu.co/index. $\mathrm{php/aquichan/article/view/1607}$

9. Santiago-Bazán C, Fernández-Cardoza J del R, Dávila-Ochoa K. Prevalencia y los factores asociados al dolor lumbar en amas de casa mayores de 18 años en el Asentamiento Humano Daniel A. Carrión del Distrito de San Martín de Porres; en el año 2007 [Internet]. [citado 2012 Oct Disponible en: http://www.efisioterapia.net/ articulos/ prevalencia-y-los-factores-asociados-al-dolor-lumbaramas-casa-mayores-18-anos-el-asentami .

10. Verbunt JA, Sieben JM, Seelen HAM, Vlaeyen JWS, Bousema EJ, van der Heijden GJ, et al. Decline in physical activity, disability and pain-related fear in sub-acute low back pain. Eur J Pain Lond Engl. agosto de 2005;9(4):417-25.

11. Sáez A. V, Troncoso Quijano C. Prevalencia, percepción de síntomas y factores de riesgo de lesiones músculo-esqueléticas en trabajadores expuestos y no expuestos a bajas temperaturas. CiencTrab. 2007 Sep;9(25):99-112.

12. Martínez MG, García IMG, Pimpinela MTC, Fuentes JM, Faz FJM. Relación entre dolor lumbar y ansiedad y su implicación terapéutica. Rev Fisioter. 2010;9(2):35-43
Recibido: 21 de Julio de 2014 Aprobado: 06 de Octubre de 2014 